\title{
Arenophile-Mediated Photochemical Dearomatization of Nonactivated Arenes
}

\author{
Mikiko Okumura ${ }^{a}$ and David Sarlah $\S^{\star a b}$
}

§Grammaticakis-Neumann Prize 2019

\begin{abstract}
Aromatic compounds are one of the most abundant classes of organic molecules and find utility as precursors for alicyclic hydrocarbon building blocks. While many established dearomatization reactions are exceptionally powerful, dearomatization with concurrent introduction of functionality, i.e. dearomative functionalization, is still a largely underdeveloped field. This review aims to provide an overview of our recent efforts and progress in the development of dearomative functionalization of simple and nonactivated arenes using arenophile-arene cycloaddition platform. These cycloadducts, formed via a visible-light-mediated [4+2]-photocycloaddition, can be elaborated in situ through olefin chemistry or transition-metal-catalyzed ring-opening with carbon-, nitrogen-, and oxygen-based nucleophiles, providing access to diverse structures with functional and stereochemical complexity. Moreover, the dearomatized products are amenable to further elaborations, which effectively install other functionalities onto the resulting alicyclic carbocycles. The utility of the arenophile-mediated dearomatization methods are also highlighted by the facile syntheses of natural products and bioactive compounds through novel disconnections.
\end{abstract}

Keywords: Arenophile $\cdot$ Catalysis $\cdot$ Dearomatization $\cdot$ Functionalization $\cdot$ Natural products

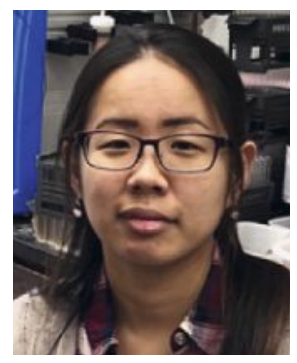

Mikiko Okumura was born and raised in Japan. She received her B.S. (2012) and M.S. (2014) in chemistry at The University of Tokyo working with Professor Shū Kobayashi. She then pursued her graduate studies in the Sarlah group at the University of Illinois at Urbana-Champaign, where she obtained her $\mathrm{PhD}$ (2019) by developing new dearomatization strategies using arenophiles. Currently she is a JSPS postdoctoral fellow in the laboratory of Professor Thomas J. Maimone at UC Berkeley.

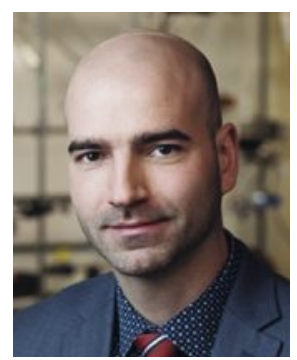

David Sarlah is an assistant professor in the Department of Chemistry at the University of Illinois at Urbana-Champaign. He was born in Slovenia, where he earned his B.S. degree from the University of Ljubljana. He obtained his $\mathrm{PhD}$ in 2011 with Professor K. C. Nicolaou at The Scripps Research Institute, and then joined the laboratory of Professor Erick M. Carreira at ETH Zürich. In 2014, David returned to the States to start his own laboratory, which explores both chemical synthesis of biologically active natural products and method development. Recently, David joined the University of Pavia as an adjunct associate professor, initiating a research program based on medicinal chemistry and drug discovery.

\section{Introduction}

Dearomatization of aromatic hydrocarbons is a fundamental synthetic strategy which provides a direct connection between readily available aromatic hydrocarbons and valuable alicyclic motifs in pharmaceuticals, agrochemicals, and natural products. ${ }^{[1]}$ Accordingly, many enabling dearomative transformations have been developed and applied to the synthesis of complex bioactive compounds. ${ }^{[2]}$ Nevertheless, most of the advancements have focused on the use of more activated arenes, such as phenols, indoles, and pyridines, and the methods to utilize simple and nonactivated arenes, such as benzene and naphthalene, are significantly limited. [3] Considering their widespread availability, the development of novel dearomative methods for nonactivated arenes would prove synthetically valuable for accessing alicyclic structures with functional and stereochemical diversity.

Cycloaddition reactions have a long and rich history for inducing dearomatization, as exemplified in UV-light-promoted arenealkene photocycloadditions. ${ }^{[4]}$ We became particularly interested in reactions reported by Sheridan, where unusual para-selective cycloaddition of benzene and naphthalene with $N$-methyl-1,2,4triazoline-3,5-dione (MTAD, 1) was observed upon visiblelight irradiation, affording arene-MTAD cycloadducts of type 2 (Scheme 1). ${ }^{[5]}$ Although these cycloadducts rapidly underwent cycloreversion at ambient temperature, their stability at lower temperatures encouraged us to exploit their reactivities toward in situ functionalization. Thus, we defined such $2 \pi$-components as arenophiles in analogy with thermal cycloaddition processes, and explored two approaches for further converting the corresponding arene-arenophile cycloadducts: olefin-like functionalization and transition-metal-catalyzed aminofunctionalization. ${ }^{[6,7]}$

\section{Olefin-like Dearomative Functionalization}

Our investigation into an arenophile-mediated dearomative functionalization began with the development of a dearomative $\mathrm{cis}$ - 


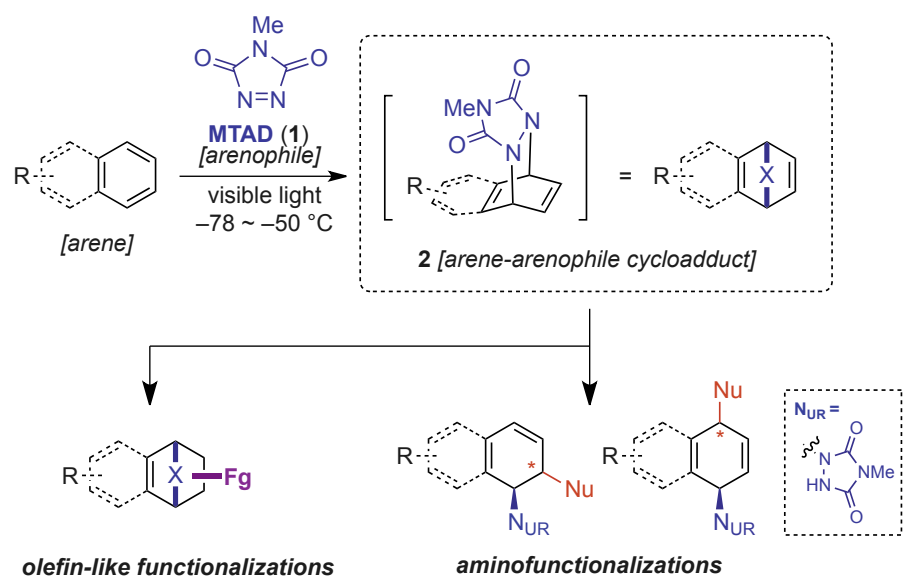

Scheme 1. Arenophile-mediated dearomative functionalization.

dihydroxylation strategy, ${ }^{[7]}$ which provided a chemical equivalent of microbial arene oxidation. ${ }^{[8]}$ In this process, cycloadducts derived from monocyclic arenes were subjected to osmium-catalyzed dihydroxylation, furnishing diols $\mathbf{3}$ with exclusive diastereoselectivity, as well as chemoselectively of the dihydroxylation at the less substituted olefin (Scheme 2A). The bicyclic diols 3, upon acetonide protection, were readily converted to dihydrodiols $\mathbf{4}$ through a one-pot arenophile cycloreversion process involving hydrolysis of urazole moiety and subsequent oxidation of bicyclic hydrazine to diazene and spontaneous extrusion of nitrogen. Alternatively, the same intermediates could be transformed into diaminohydrodiols 5 via arenophile fragmentation in a two-step protocol: one-pot urazole hydrolysis and bis-benzoylation of the resulting hydrazine, and a reductive $\mathrm{N}-\mathrm{N}$ cleavage using $\mathrm{SmI}_{2}$. A wide variety of functional groups were tolerated in both processes, such as alkyl, benzylic heteroatoms, halogens, protected carbonyl groups, and silyl groups. Importantly, polycyclic arenes, such as naphthalene derivatives, heteroarenes, and phenanthrene, were also viable substrates for this dearomative dihydroxylation, providing diols 6 . Similarly to the protocol for monocyclic arene-derived products, subsequent arenophile fragmentation furnished diaminodiols 7. In all cases, the cycloaddition reaction proceeded with exclusive siteselectivity at the terminal non-substituted benzenoid ring.

We envisioned application of the dearomative dihydroxylation protocol with bromobenzene $(\mathbf{8})$ towards the syntheses of Amaryllidaceae alkaloids lycoricidine (12) and narciclasine (13). [9] As shown in Scheme 2B, Narasaka-Sharpless modification of the Upjohn protocol provided the dihydroxylated product 9 in a form of boronic ester, which was readily subjected to a palladium-catalyzed transpositive Suzuki coupling reaction with the vinyl bromide and the arylboronic ester moiety to construct the key $\mathrm{C}-\mathrm{C}$ bond (10). ${ }^{[10]}$ Further transformation into the isocarbostyril common framework $\mathbf{1 1}$ was achieved in a three-step sequence, and then provided lycoricidine (12) and narciclasine (13), respectively.

Based on a similar design, a dearomative epoxidation was achieved through a manganese-catalyzed epoxidation (Scheme 3A). ${ }^{[11]}$ This process worked well for both monocyclic and polycyclic arenes, as well as for heteroarenes, without any formation of the corresponding $N$-oxides. The resulting bicyclic epoxides 14 were key intermediates in accessing arene oxides $\mathbf{1 5}$ and oxepines $\mathbf{1 6 .}$ Such compounds are common metabolites, produced by $\mathrm{P} 450$ based monooxygenases in eukaryotes; however, their chemical accessibility has been significantly hampered due to their susceptibility towards the NIH-shift. ${ }^{[12]}$ Through arenophile cycloreversion under base-induced conditions and subsequent oxidation, a range of cycloadducts $\mathbf{1 4}$ derived from monocyclic and polycyclic arenes afforded arene oxides $\mathbf{1 5}$ and oxepines 16, respectively. ${ }^{[13]}$ Naphthalene-derived oxepine $\mathbf{1 7}$ could be further elaborated to incorporate more functionalities, as exemplified in Scheme 3B.
Scheme 2. Dearomative dihydroxylation. (A) General scheme and representative scope. (B) Synthesis of lycoricidine (12) and narciclasine (13).

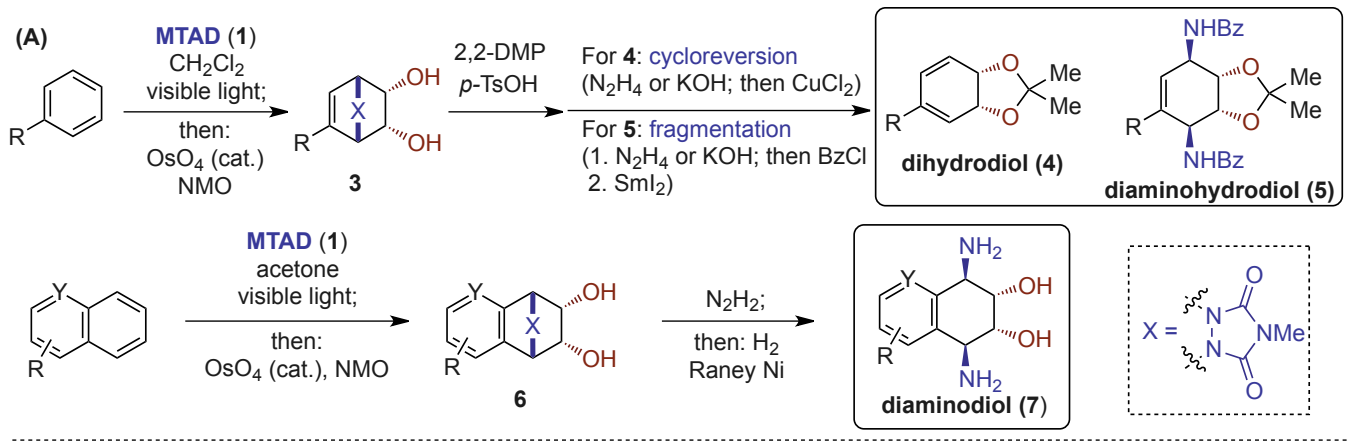

dihydrodiol (4)

(14\% 39\% yield over three steps)

diaminohydrodiol (5)

(13\% 32\% yield over four steps)

(21\% 63\% yield over two steps)

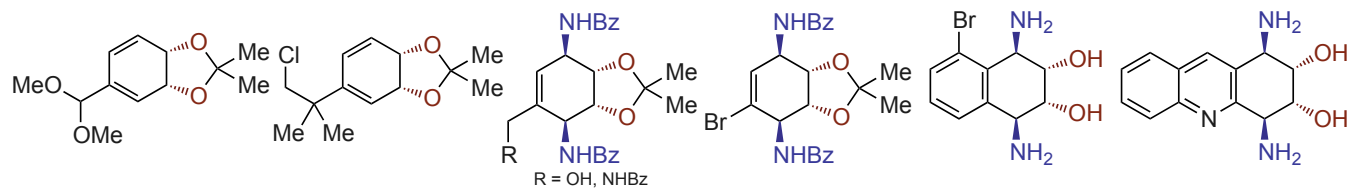

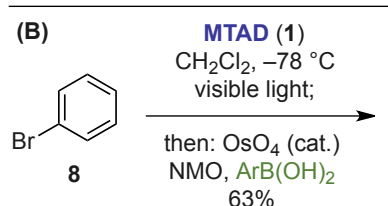

$63 \%$

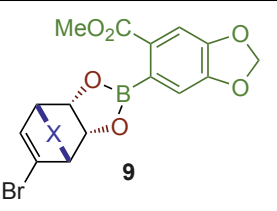

$\mathrm{Pd}(\mathrm{dppf}) \mathrm{Cl}_{2}$
(cat.)

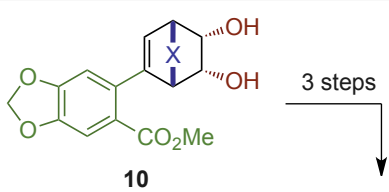

10

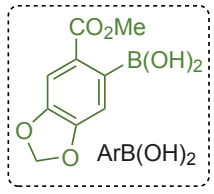

(士)-lycoricidine (12)

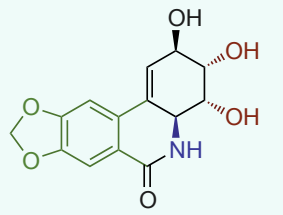

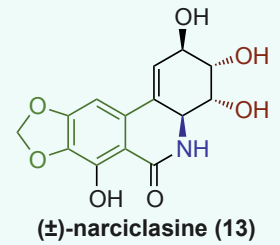

(士)-narciclasine (13)

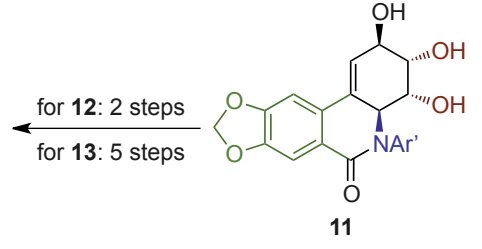




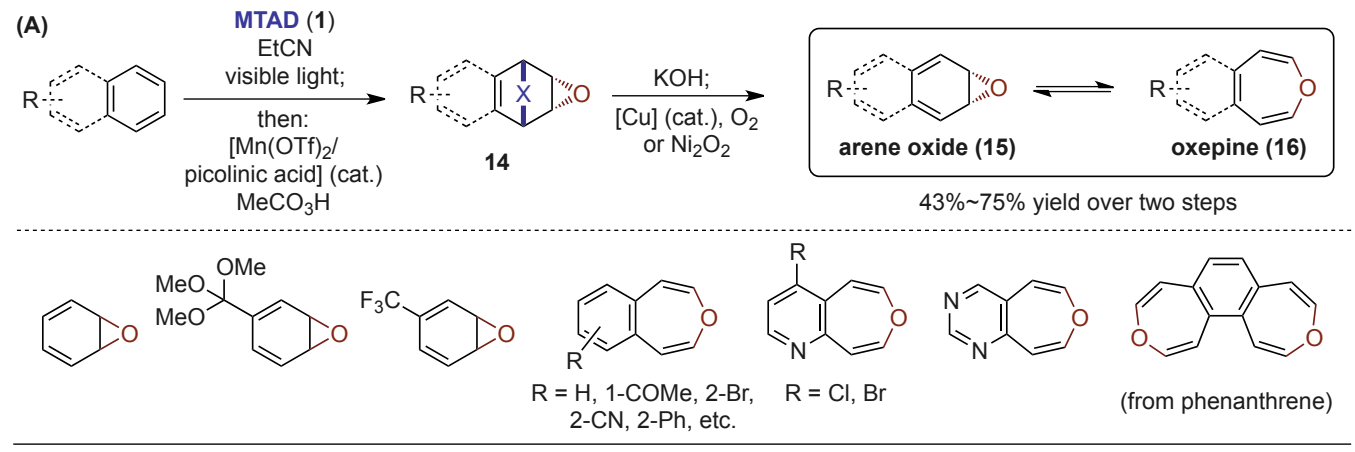

Scheme 3. Dearomative epoxidation. (A) General scheme and representative scope. (B) Oxepine derivatizations.

(B)

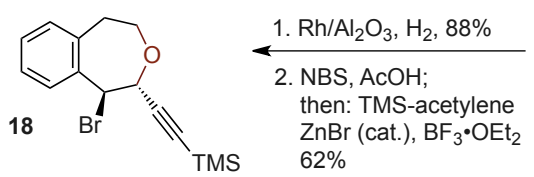

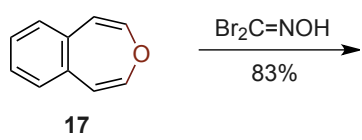<smiles>OC12ON=C(Br)C1OC=Cc1ccccc12</smiles>

In contrast, the reductive conversion of the arene-arenophile cycloadducts was conducted using in situ generated diimide to provide saturated cycloadducts of type 20 (Scheme 4A). ${ }^{[14]}$ For example, monocyclic arene-derived products $\mathbf{2 0}$ were manipulated into 1,3-cyclohexadienes $\mathbf{2 1}$ and diaminocyclohexenes $\mathbf{2 2}$ through cycloreversion or fragmentation of urazole moiety. On the other hand, the polycyclic arenes undergo formal bis-1,4-hydroamination via two one-pot sequences: dearomative reduction, followed by hydrolysis of arenophile moiety and reductive $\mathrm{N}-\mathrm{N}$ cleavage $(\mathbf{2 3} \rightarrow \mathbf{2 4}$, Scheme 4B). Additionally, naphthalene-derived reduced cycloadduct was readily converted to aminoalcohol $\mathbf{2 5}$ and the natural product $\gamma$-hydroxytetralone $\mathbf{2 6}$.

Finally, we recently reported the application of arenophilemediated dearomative hydroboration as the key step in the concise synthesis of idarubicinone $(\mathbf{3 3}) .^{[15,16]}$ Thus, upon oxidative conversion of tetracene to tetracenequinone 27 , rhodium-catalyzed dearomative hydroboration with catecholborane, and subsequent transesterification provided pinacol boronic ester $\mathbf{2 8}$ on a gram scale (Scheme 5). Zweifel olefination with subsequent hydrolysis of the resulting enol ether moiety revealed the requisite methyl ketone 29, and subsequent $\beta$-elimination of the urazole $(\mathbf{2 9} \rightarrow \mathbf{3 0})$ set the stage for $\alpha$-oxidation through hydrogen atom transfer reaction to form hydroxyketone 31. Finally, methyl ether deprotection $(\mathbf{3 1} \rightarrow \mathbf{3 2})$ and redox exchange of the urazole to the hydroxy group completed the synthesis of $( \pm)$-idarubicinone (33).

\section{Transition-Metal-catalyzed Dearomative Aminofunctionalization}

While olefin functionalizations of arene-arenophile cycloadducts proved useful in providing a rapid and controlled access to a wide variety of highly functionalized alicyclic motifs, we explored additional reactivities to further extend the utility of arenophile-based dearomatizations. Specifically, we investigated if arenophile-arene cycloadducts were amenable to ring-opening reactions, as similar processes are well known for structurally related azabenzonorbornadienes. ${ }^{[17]}$ Indeed, we discovered that the arene-arenophile bicycles are viable substrates for transitionmetal-catalyzed ring-openings, and the resulting organometallic intermediates could be captured with various nucleophiles, affording a diverse range of aminofunctionalized products.

One of the first successful approaches discovered in this process was Ni-catalyzed carboamination with Grignard reagents as nucleophiles, delivering products with exclusive trans-1,2selectivity (34, Scheme 6A) ${ }^{[18]}$ Aryl and vinyl Grignards were applied to this procedure, and the reaction delivered carboaminated products in high yields as well as high enantioselectivity for benzene and naphthalene using P,N-bidentate ligand 35.[18b] The catalytic cycle likely proceeds through an initial $\pi$-complexation and formation of $\eta^{5}$-nickel cyclohexadienyl intermediate $(\mathbf{I} \rightarrow \mathbf{I I})$, which then undergoes transmetalation $($ II $\rightarrow$ III), followed by inner-sphere reductive elimination $(\mathbf{I I I} \rightarrow \mathbf{I V})$ to deliver trans-1,2carboaminated product (Scheme 7).[18a]
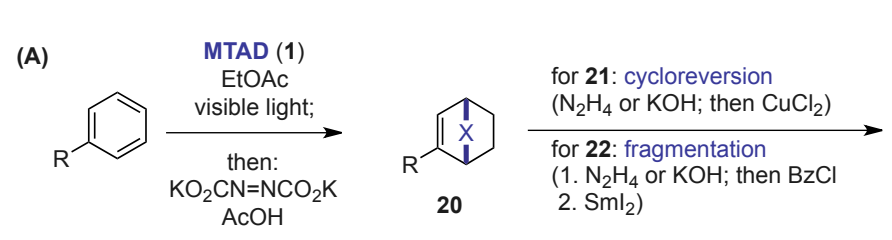
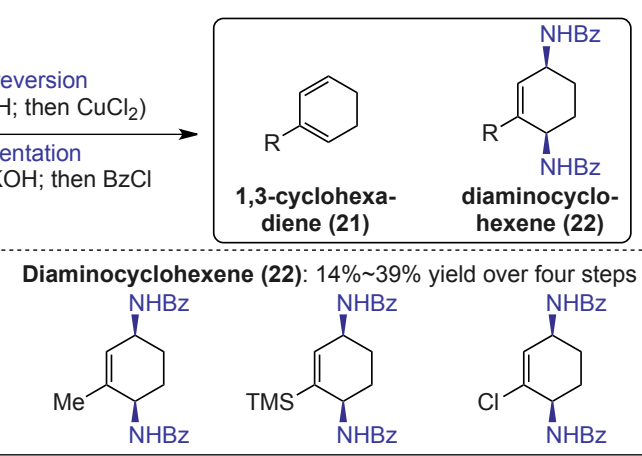

1,3-cyclohexadiene (21): $35 \% \sim 67 \%$ yield over two steps
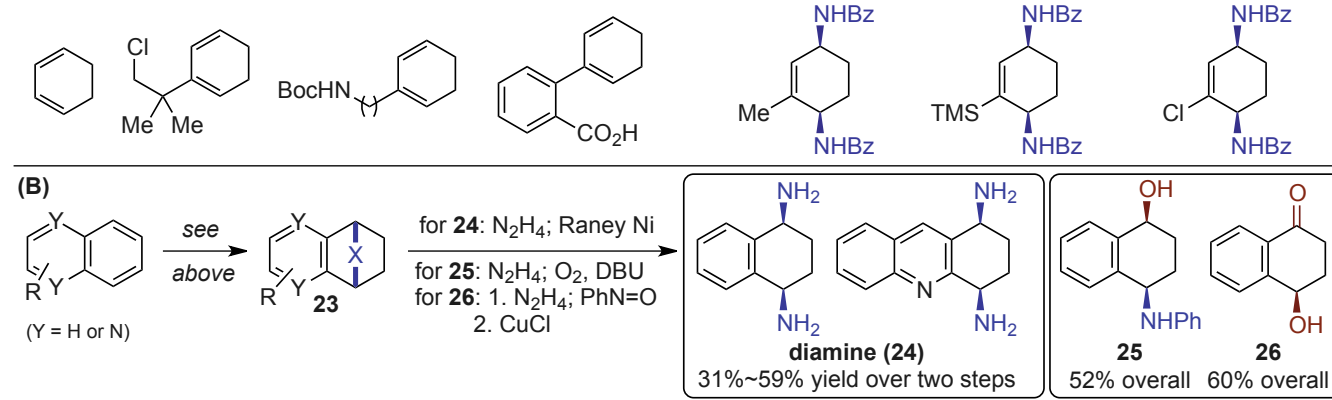

Scheme 4. Dearomative reduction. (A) General scheme for mononuclear arenes and representative scope. (B) General scheme for polynuclear arenes and representative scope. 
Scheme 5. Synthesis of idarubicinone (33) through arenophile-mediated dearomative hydroboration.
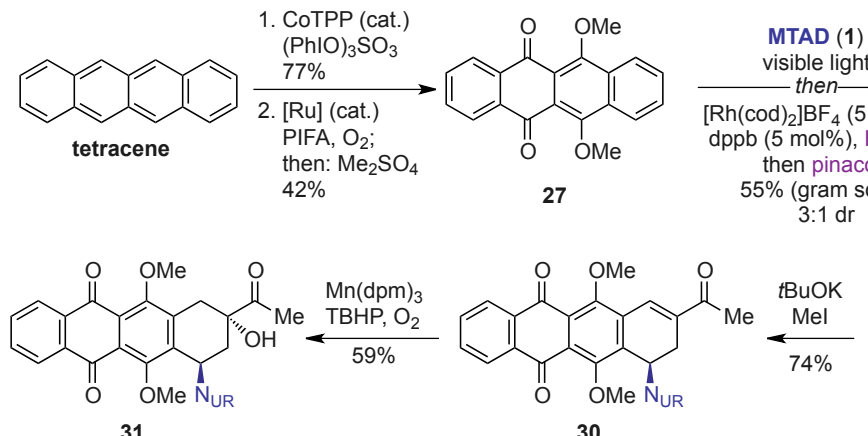<smiles>COc1c2c(c(OC)c3c1C1CCC3C(Br)C1)C(=O)c1ccccc1C2=O</smiles>
$\mathrm{Zn}, \mathrm{TMSCl}$ i. OEt $\lambda_{L i}$ iii. $\mathrm{I}_{2}, \mathrm{NaOMe}$<smiles>COC1=C2c3c(c(OC)c4c(c3OC)C(=O)c3ccccc3C4=O)CC1(O)C[C@@H]2N[13C](=O)[18OH]</smiles>

31<smiles>C=NC1CC(C(C)=O)=Cc2c(OC)c3c(c(OC)c21)C(=O)c1ccccc1C3=O</smiles><smiles>COc1c2c(c(OC)c3c1C1CC3C(=O)c3ccccc3C1=O)C1CC2C[C@H]1C(C)=O</smiles>
$72 \%$

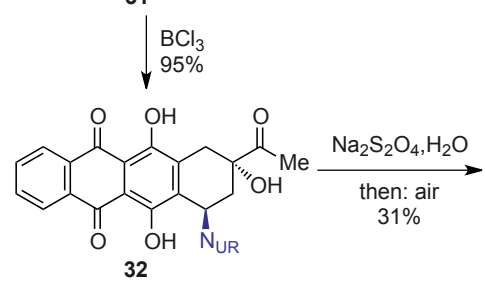<smiles>CC(=O)[C@]1(O)Cc2c(O)c3c(c(O)c2[C@@H](O)C1)C(=O)c1ccccc1C3=O</smiles>

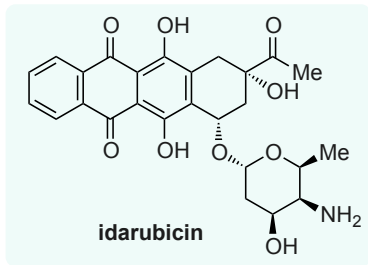

Scheme 6. Ni-catalyzed dearomative trans-1,2-carboamination. (A) General scheme and representative scope. (B) Synthesis of isocarbostyril alkaloids and analogs.
(A)
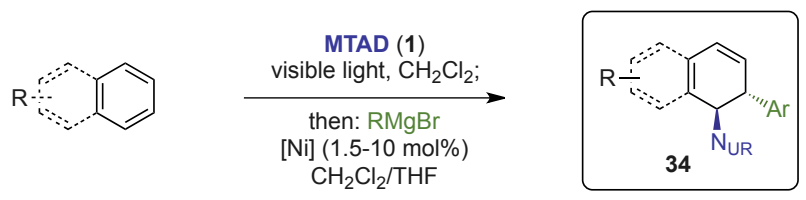

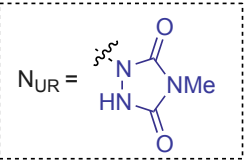

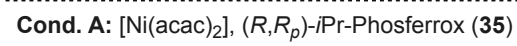

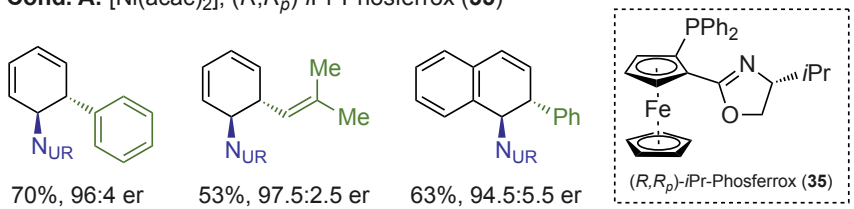

Cond. B: Ni(cod) $)_{2}$, dppf. *Ratio of constitutional isomers. $\overbrace{\mathrm{N}_{U R}}^{(\mathrm{Ph}}$

(B)

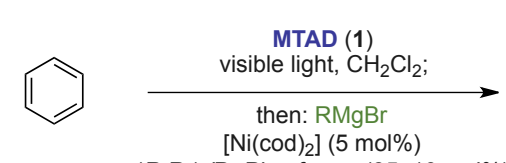

$\left(R, R_{p}\right)$-iPr-Phosferrox $(35,10 \mathrm{~mol} \%)$

then: $\mathrm{Me}_{2} \mathrm{SO}_{4}$

$65 \%$ (98:2 er)

[>10g scale]<smiles>O=C1N[C@H]2c3c(cc4c(c3O)OCO4)[C@@H]3[C@@H](O)[C@H](O)[C@@H](O)[C@@H](O)[C@@H]3NC12</smiles>

(+)-pancratistatin (40)
$62 \%$

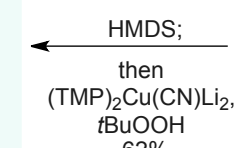<smiles>NC1C=CC=CC1c1ccc2c(c1)OCO2</smiles>

36

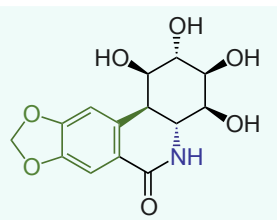

(+)-7-deoxypancratistatin (39)

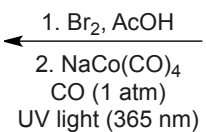

1. $\mathrm{Br}_{2}, \mathrm{AcOH}$
2. $\mathrm{NaCo}(\mathrm{CO})_{4}$
$\mathrm{CO}(1 \mathrm{~atm})$
UV light $(365 \mathrm{~nm})$
$72 \%$ overall

1. $\mathrm{Br}_{2}, \mathrm{AcOH}$
2. $\mathrm{NaCo}(\mathrm{CO})_{4}$
$\mathrm{CO}(1 \mathrm{~atm})$
UV light $(365 \mathrm{~nm})$
$72 \%$ overall

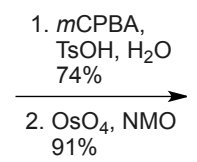

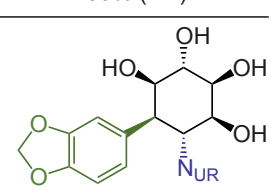

37

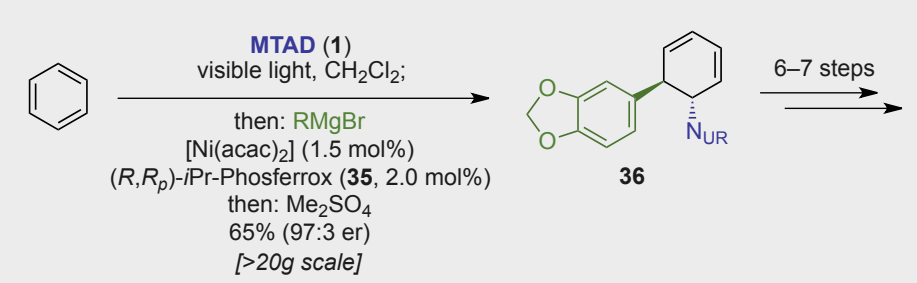

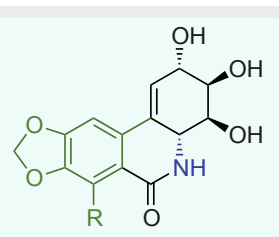

(+)-lycoricidine $(12, \mathrm{R}=\mathrm{H})$ $(+)$-narciclasine $(13, \mathrm{R}=\mathrm{OH})$
The Ni-catalyzed dearomative carboamination was applied towards the enantioselective total synthesis of isocarbostyril alkaloids from benzene, as this process enabled the construction of the core carbon skeleton in a single step, as well as installed the requisite nitrogen- and carbon-based substituents on the aminocyclitol core with desired stereochemistry. The synthesis of (+)-7-deoxypancratistatin (39) was achieved from the diene $\mathbf{3 6}$ through two oxidation steps $(\mathbf{3 6} \rightarrow \mathbf{3 7})$, urazole reduction to amine $\mathbf{3 8}$, and subsequent Co-catalyzed carbonylative coupling to form the lactam (Scheme 6B). ${ }^{[18 a, 19]}$ The direct conversion of (+)-7-deoxypancra- tistatin (39) to (+)-pancratistatin (40) was also realized in a single step through formal C-H oxidation. Similarly, the enantioselective synthesis of (+)-lycoricidine (12) and (+)-narciclasine (13) was achieved in 6-7 steps from the same diene intermediate 36. ${ }^{[19]}$

Interestingly, using palladium as a catalyst, we found that the selectivity of the dearomative carboamination with Grignard reagents occurred exclusively with syn-1,4-selectivity. ${ }^{[20]}$ The reaction with benzene proceeded smoothly with a wide variety of aryl, vinyl, and alkyl Grignard reagents, and the scope of the arenes was extended to various monocyclic and polycyclic arenes (Scheme 


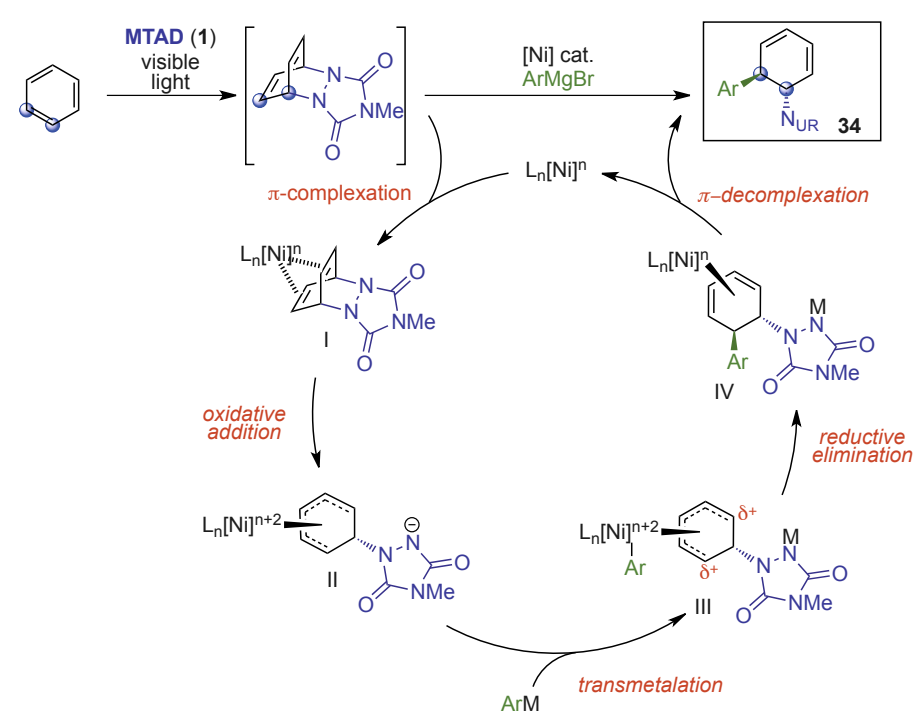

Scheme 7. Plausible mechanism for Ni-catalyzed dearomative trans-1,2carboamination.

$8 \mathrm{~A}$, conditions $\mathrm{A}$ and $\mathrm{B})$. The synthetic utility of this process was demonstrated by the preparation of Sertraline (47), ${ }^{[21]}$ one of the most prescribed antidepressants, from naphthalene (Scheme
8B). Thus, the dearomative syn-1,4-carboamination (naphthalene $\rightarrow \mathbf{4 5}$ ) introduced all the handles with necessary stereochemical syn-1,4-relationship in a single dearomative transformation. Upon olefin hydrogenation, urazole hydrolysis, and subsequent reduction, Sertraline (47) was obtained in four overall operations. Moreover, we established an enantioselective variant of the syn-1,4-carboamination using (S)-DIFLUORPHOS, delivering high enantioselectivity when the reaction was conducted with an isolated naphthalene-MTAD cycloadduct (48).

In addition to Grignard reagents, ketone- and ester-derived lithium-enolates were also viable carbon-based nucleophiles for this type of dearomative syn-1,4-carboamination (Scheme 8A, conditions C-E). [22] Notably, more sterically encumbered $\alpha$-branched carbonyl nucleophiles had little effect on the reaction outcome, and an enantioselective variant gave high selectivity in the case of naphthalene. Mechanistically, these reactions are presumed to proceed through $\pi$-allyl palladium species with outersphere attack of nucleophiles.

We also found that the heteroatom-based nucleophiles are compatible with Pd-catalyzed dearomative syn-1,4-aminofunctionalizations (49, Scheme 9). For instance, with naphthalene as a substrate, both primary and secondary amines coupled smoothly with MTAD-arene cycloadducts (conditions A for primary amine and B for secondary amine). ${ }^{[23]}$ This transformation was also efficient for substituted naphthalenes, heteroarenes, and
(A)

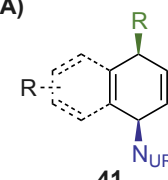

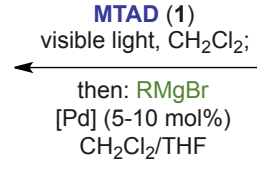

41
$\mathrm{CH}_{2} \mathrm{Cl}_{2} / \mathrm{THF}$

Cond. A: Pd(dba $)_{2}$, DPEphos Cond. B: $\mathrm{Pd}(\mathrm{dba})_{2}, \mathrm{P}\left(\mathrm{o}-\mathrm{MeC}_{6} \mathrm{H}_{4}\right)_{3}$ ${ }^{*}$ Ratio of constitutional isomers.

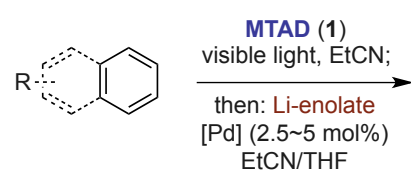

Cond. C: $\mathrm{Pd}(\mathrm{dba})_{2}, \mathrm{dppb}$ Cond. D: $\mathrm{Pd}(\mathrm{dba})_{2},\left(\mathrm{~S}, \mathrm{~S}_{\mathrm{p}}\right)$-tBu-Phosferrox $(43)$ Cond. E: $\left[\mathrm{Pd}(\right.$ allyl $) \mathrm{Cl}_{2},(R)$-DTBM-SEGPHOS (44)

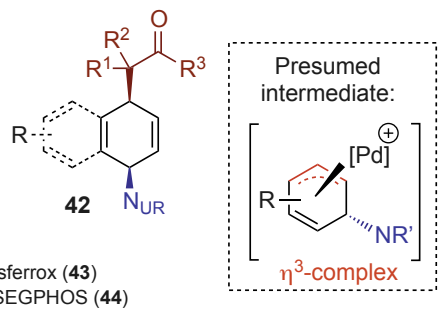<smiles>NC1C=CC(CC(=O)C2CCCCC2)c2ccccc21</smiles><smiles>COC(=O)C(C)(C)C1C=CC(N)c2ccccc21</smiles>

Cond. C: $72 \%$ Cond. E: $61 \%$

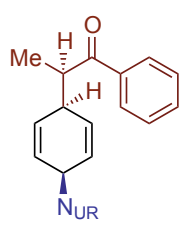

Cond. C: $86 \%$ $\begin{array}{rll}\text { Cond. A: } 50 \% \text { Cond. B: } 71 \% & \text { Cond. B: } 56 \% \text { Cond. B: } 72 \% & \text { Cond. C: } 87 \% \\ \text { Cond. D: } 68 \%\end{array}$

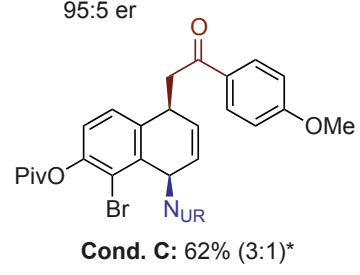

Cond. B: $47 \%(1.3: 1)^{*}$
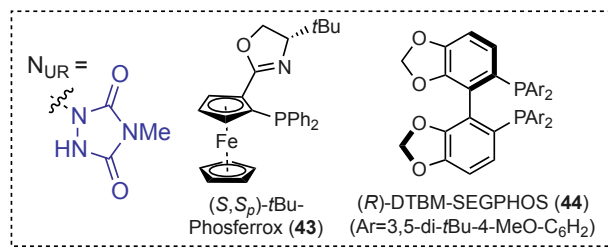

(B)

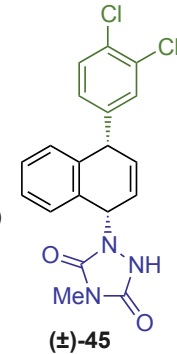

1. $\mathrm{H}_{2}(1 \mathrm{~atm})$

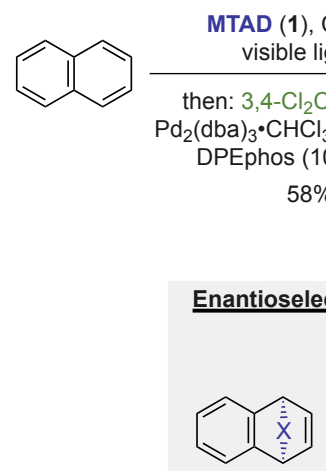

48
$\mathrm{Rh} / \mathrm{Al}_{2} \mathrm{O}_{3}$ (cat.) $\mathrm{Rh} / \mathrm{Al}_{2} \mathrm{O}$
$98 \%$

2. $\mathrm{B}$

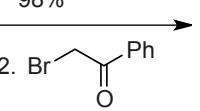

$\mathrm{NaOEt}$ then: $\mathrm{KOH}, \Delta$ $51 \%$<smiles>NCc1cc[c-]cc1CCCCCCO</smiles>

(士)-46

Scheme 8. Pd-catalyzed dearomative syn-1,4-carboamination. (A) General scheme and representative scope. (B) Synthesis of Sertraline (47). 
benzene. Moreover, oxygen-based nucleophiles such as benzyl alcohols and oximes worked well to provide the syn-1,4-oxyaminated products (50, conditions D). ${ }^{[24]}$ The enantioselective version was developed for naphthalene-derived cycloadduct using both nitrogen- and oxygen-based nucleophiles, and notably syn-1,4-diaminated and syn-1,4-oxyaminated compounds were obtained with high enantioselectivity (conditions $\mathrm{C}$ and $\mathrm{E}$ ). Finally, the dearomative diamination protocol was applied to the structural elaboration of memantine, an FDA-approved drug used for the treatment of dementia associated with Alzheimer's disease. Successive chemoselective hydrogenation of the olefin in 52, followed by oxidation of the urazole moiety afforded aminoketone $\mathbf{5 3}$.

\section{Conclusion and Outlook}

In conclusion, we have developed dearomative functionalization methods for simple arenes, which are conceptually distinct from other established dearomatizations, providing rapid entry to a variety of complex alicyclic structures. The salient features of these transformations are: 1) dearomatization under mild conditions (visible-light irradiation, low temperature), permitting the use of sensitive functional groups, such as halogens and benzylic heteroatom functional groups, which are traditionally not tolerated during standard dearomative events; 2) a broad scope of amenable arene substrates, including mono- and polycyclic arenes; 3 ) complementary site-selectivity for the dearomatization of polycyclic arenes and heteroarenes; 4) installation of a wide variety of functionality with well-defined stereochemistry; 5) high enantioselectivity of dearomatized products; and 6) empowering novel disconnections towards the facile synthesis of natural products and bioactive compounds from simple arenes. Importantly, most of the products obtained through arenophile-based methods are orthogonal to established dearomative transformations, including biocatalysis.

\section{Acknowledgments}

We would like to acknowledge the University of Illinois, Petroleum Research Fund (PRF\#57175-DNI1), the National Science Foundation (CAREER Award No. CHE-1654110), and the NIH/National Institute of General Medical Sciences (R01 GM122891) for the support to carry the described research. We also thanks Amgen, Bristol-Myers Squibb, Eli Lilly, and FMC for unrestricted research support. D.S. is an Alfred P. Sloan Fellow. M.O. acknowledges the Honjo International Scholarship Foundation.

Received: July 5, 2020

[1] a) F. Foubelo, M. Yus in 'Arene Chemistry: Reaction Mechanisms and Methods for Aromatic Compounds', Eds. J. Mortier, John Wiley-VCH Weinheim, 2015; b) 'Asymmetric Dearomatization Reactions', Ed. S.-L. You, Wiley-VCH, 2016.

[2] S. P. Roche, J. A. Porco Jr., Angew. Chem. Int. Ed. 2011, 50, 4068.

[3] a) C.-X. Zhuo, W. Zhang, S.-L. You, Angew. Chem. Int. Ed. 2012, 51, 12662; b) C. Zheng, S.-L. You, Chem. 2016, 1, 830; c) W. C. Wertjes, E. H. Southgate, D. Sarlah, Chem. Soc. Rev. 2018, 47, 7996; d) M. Okumura, D. Sarlah, Eur. J. Org. Chem. 2020, 10, 1259.

[4] a) R. Remy, C. G. Bochet, Chem. Rev. 2016, 116, 9816; b) U. Streit, C. G. Bochet, Beilstein J. Org. Chem. 2011, 7, 525.

[5] a) D. P. Kjell, R. S. Sheridan, J. Am. Chem. Soc. 1984, 106, 5368; b) S. V. Hamrock, R. S. Sheridan, J. Am. Chem. Soc. 1989, 111, 9247.

[6] M. Okumura, D. Sarlah, Synlett 2018, 29, 845.

[7] E. H. Southgate, J. Pospech, J. Fu, D. R. Holycross, D. Sarlah, Nat. Chem. 2016, 8,922

[8] R. A. Johnson, Org. React. 2004, 63, 117.

[9] S. Ghosal, S. K. Singh, Y. Kumar, R. S. Srivastava, Phytochemistry 1989, 28, 611.

[10] E. H. Southgate, D. R. Holycross, D. Sarlah, Angew. Chem. Int. Ed. 2017, $56,15049$.

[11] Z. Siddiqi, W. C. Wertjes, E. H. Southgate, D. Sarlah, J. Am. Chem. Soc. 2020, 142, 10125 .

[12] a) G. J. Kasperek, T. C. Bruice, J. Am. Chem. Soc. 1972, 94, 198; b) T. C. Bruice, P. Y. Bruice, Acc. Chem. Res. 1976, 9, 378; c) G. Guroff, J. Renson, S. Udenfriend, J. W. Daly, D. M. Jerina, B. Witkop, Science 1967, 157, 1524; b) D. M. Jerina, J. W. Daly, Science 1974, 185, 573.
Scheme 9. Pd-catalyzed dearomative syn-1,4-diamination and oxyamination. (A) General scheme and representative scope. (B) Derivatizations.
(A)
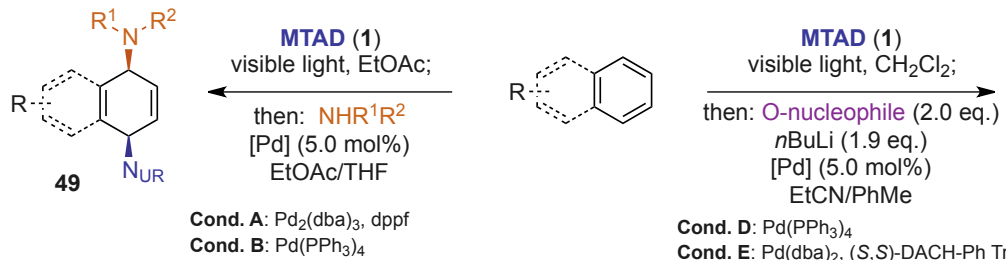

Cond. D: $\mathrm{Pd}\left(\mathrm{PPh}_{3}\right)_{4}$

Cond. E: Pd(dba) $)_{2},(S, S)$-DACH-Ph Trost Ligand (51)

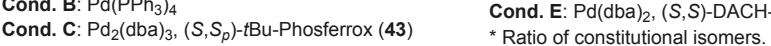
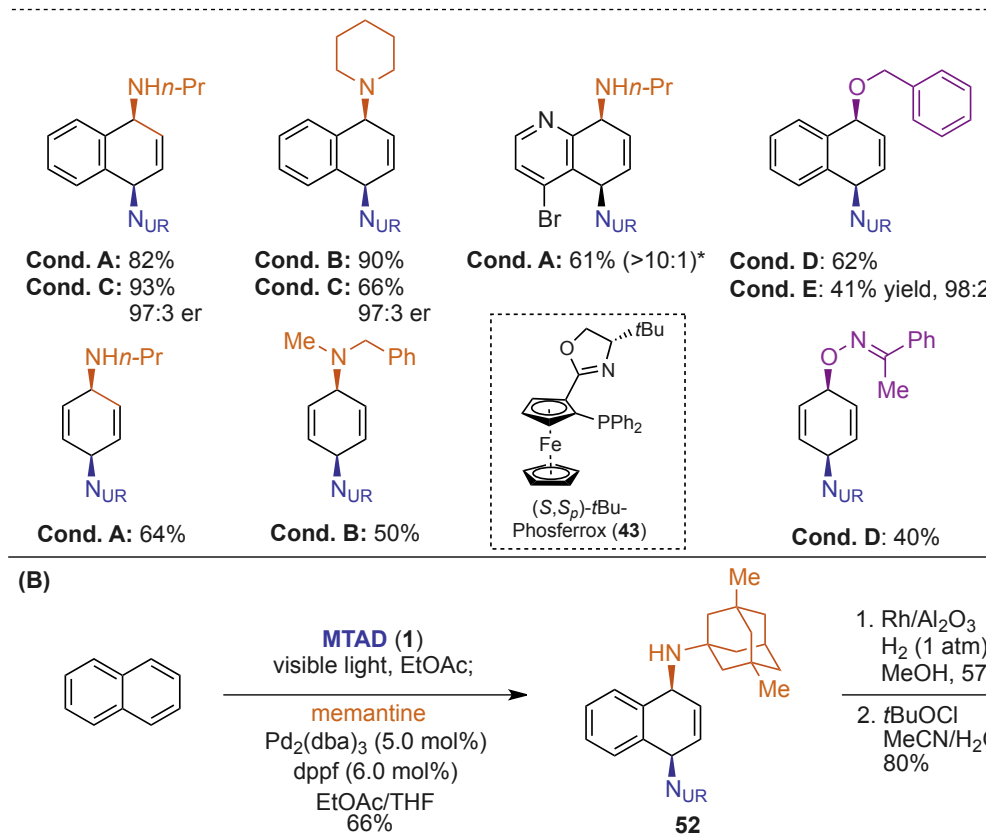

Cond. D: $62 \%$
Cond. E: $41 \%$ yield, $98: 2$ er

Cond. D: $70 \%$

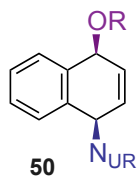

50

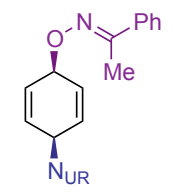

Cond. D: $40 \%$

(2)
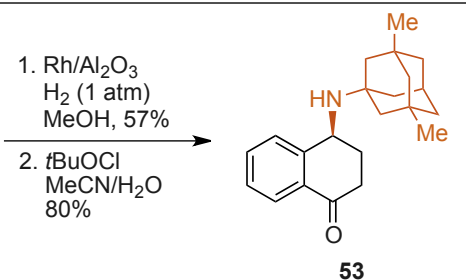
[13] a) E. Vogel, H. Günther, Angew. Chem. Int. Ed. Engl. 1967, 6, 385; b) D. R. Boyd, N. D. Sharma, Chem. Soc. Rev. 1996, 25, 289.

[14] M. Okumura, S. M. Nakamata Huynh, J. Pospech, D. Sarlah, Angew. Chem. Int. Ed. 2016, 55, 15910 .

[15] a) L. M. Hollingshead, D. Faulds, Drugs 1991, 42, 690; b) R. J. Cersosimo, Clin. Pharm. 1992, 11, 152.

[16] D. G. Dennis, M. Okumura, D. Sarlah, J. Am. Chem. Soc. 2019, 141, 10193.

[17] a) M. Lautens, K. Fagnou, S. Hiebert, Acc. Chem. Res. 2003, 36, 48; b) D. K. Rayabarapu, C.-H. Cheng, Acc. Chem. Res. 2007, 40, 971; (c) S. Woo, B. A. Keay, Synthesis 1996, 1996, 669; d) M. Lautens, P. Chiu, Top. Curr. Chem. 1997, 190, 1 .

[18] a) L. W. Hernandez, J. Pospech, U. Klöeckner, T. W. Bingham, D. Sarlah J. Am. Chem. Soc. 2017, 139, 15656; b) L. W. Hernandez, U. Klöckner, J. Pospech, L. Hauss, D. Sarlah, J. Am. Chem. Soc. 2018, 140, 4503.

[19] T. W. Bingham, L. W. Hernandez, D. G. Olson, R. L. Svec, P. J. Hergenrother, D. Sarlah, J. Am. Chem. Soc. 2019, 141, 657.

[20] C. Tang, M. Okumura, Y. Zhu, A. Hooper, Y. Zhou, Y. Lee, D. Sarlah, Angew. Chem. Int. Ed. 2019, 58, 10245.

[21] W. M. Welch, A. R. Kraska, R. Sarges, B. K. Koe, J. Med. Chem. 1984, 27, 1508.

[22] M. Okumura, A. S. Shved, D. Sarlah, J. Am. Chem. Soc. 2017, 139, 17787.

[23] W. C. Wertjes, M. Okumura, D. Sarlah, J. Am. Chem. Soc. 2019, 141, 163.

[24] C. Tang, M. Okumura, H. Deng, D. Sarlah, Angew. Chem. Int. Ed. 2019, 58, 15762.
License and Terms

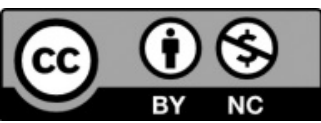

This is an Open Access article under the terms of the Creative Commons Attribution License CC BY_NC 4.0. The material may not be used for commercial purposes.

The license is subject to the CHIMIA terms and conditions: (http:// chimia.ch/component/sppagebuilder/?view=page \&id=12).

The definitive version of this article is the electronic one that can be found at doi:10.2533/chimia.2020.577 